\title{
A quantitative study of the neurofibrillary tangles and the choline acetyltransferase activity in the cerebral cortex and the amygdala in Alzheimer's disease
}

\author{
Margaret M Esiri, R C A Pearson, Janet E Steele, D M Bowen, T P S Powell
}

\begin{abstract}
A quantitative study has been made of the number of neurofibrillary tangles and of the choline acetyltransferase activity in several sites in the cerebral hemispheres of eight patients who had had Alzheimer's disease. The neurofibrillary tangles were maximal in structures in the medial temporal lobe (uncus, amygdala, hippocampus and parahippocampal gyrus), severe in the neocortex on the lateral surface of the temporal lobe, moderate in the "association cortex" of the parietal and frontal lobes and minimal in primary somatic and visual sensory areas. There was a significant decrease in choline acetyltransferase activity in almost all areas, and the means of the percentage decreases for the different groups of areas correlate well with the counts of the neurofibrillary tangles. These results support the hypothesis that the pathological process in Alzheimer's disease may spread along a sequence of corticocortical connections between the main sensory areas and the hippocampal formation. The disease process may also spread along the reciprocal connections between the amygdala and the neocortex because the numbers of tangles in different areas of the neocortex closely parallel the density of their connections and the amygdala.
\end{abstract}

Department of Neuropathology,
Radcliffe Infirmary, Oxford

M Msiri

Department of Anatomy, St Mary's

Hospital Medical

School, London

R C A Pearson

Department of

Neurochemistry, Institute of Neurology,

London

J E Steele

DM Bowen

Department of

Human Anatomy,

Oxford

T P S Powel

Correspondence to: T P S Powell, Department of Human Anatomy, South Parks Road, Oxford OX1 3QX, United Kingdom.

Received 16 March 1989 and in revised form 6 June 1989. Accepted 14 July 1989

In Alzheimer's disease the pathological uniformly, but instead the "association" cortex of the parieto-temporal and frontal lobes and the cingulate cortex are most severely affected. The motor cortex and the major sensory areas are little if at all involved. The olfactory system appears to be unique in being the only sensory system that is affected and structures associated with it also show the greatest degree of pathological change. ${ }^{1}$ On the basis of this distribution of the pathology and of the clinical features there is the possibility that the olfactory system may be the initial site of involvement and that the disease process spreads along certain cortico-cortical fibre connections. ${ }^{2}$ The parts of the neocortex that are affected are homologues of those that have been shown in the monkey to be connected by a sequence of links which starts in the main sensory areas and passes through the "association" cortex of the parieto-temporal and frontal lobes to the hippocampal formation. ${ }^{3}$ Each successive step in this orderly sequence through the parieto-temporal lobe is reciprocally connected both with the preceding step and with the corresponding step in the frontal lobe. In addition, the hippocampal formation and adjoining areas of cortex send fibres back to a number of these association areas. ${ }^{45}$ The least affected areas in Alzheimer's disease are at the beginning of this sequence, the most severe at the end and the "association" areas occupy an intermediate position and are affected to an intermediate degree.

It is possible that the distribution in the neocortex itself may also depend in part upon its connections with the amygdala. The cortico-medial group of amygdaloid nuclei receives fibres directly from the olfactory bulb ${ }^{6}$ and in turn are interconnected with the basolateral group. ${ }^{7-9}$ The latter nuclei are reciprocally connected with widespread areas of the neocortex ${ }^{1011}$ and it is significant that in the monkey the heaviest connections are with those areas that are the homologues of the regions most severely affected in Alzheimer's disease and the least connections are with the motor and sensory areas that are least affected by the pathological changes. As well as through the sequence of cortico-cortical fibres between areas of the neocortex, the disease process may spread along these connections between the amygdala and the neocortex.

These hypotheses cannot be tested directly changes in the neocortex are not distributed because it is not possible to undertake repeated examination of the same area of the brain during the course of the disease. If the hypotheses are correct, however, it is likely that no area of cortex that is only indirectly linked to the hippocampal formation by cortico-cortical connections will be affected in Alzheimer's disease. That is, unless intervening areas with relaying neurons more closely situated to the hippocampal formation are also affected. Furthermore, the more closely these areas are situated to the hippocampal formation and to the olfactory structures, and the stronger the connection to the amygdala, the more severe would be their involvement by the disease. The present quantitative study on the distribution of neurofibrillary tangles has been undertaken to test these predictions. 
Table Numbers of neurofibrillary tangles $/ \mathrm{mm}^{3}$

\begin{tabular}{|c|c|c|c|c|c|c|c|c|c|c|c|c|c|c|c|c|c|}
\hline \multirow[b]{3}{*}{ Sex } & \multirow[b]{3}{*}{ Age } & & & & & & \multicolumn{9}{|c|}{ Association cortex } & & \\
\hline & & \multicolumn{5}{|c|}{ Olfactory structures and hippocampal formation } & \multicolumn{4}{|c|}{ Temporal lobe } & \multicolumn{5}{|c|}{ Parietal and frontal lobes } & \multicolumn{2}{|c|}{ Sensory cortex } \\
\hline & & Uncus & Amyg & Hipp & Ent cortex & $T H / T F$ & 20 & 21 & 22 & 38 & 7 & 46 & 9 & 13 & 25 & $S 1$ & 17 \\
\hline $\begin{array}{l}M \\
F \\
M \\
F \\
M \\
M \\
F \\
M\end{array}$ & $\begin{array}{l}70 \\
78 \\
75 \\
73 \\
70 \\
79 \\
70 \\
75\end{array}$ & $\begin{array}{r}4200 \\
1500 \\
3440 \\
\\
3420 \\
2000 \\
830 \\
100 \\
2210 \\
\text { Mean }\end{array}$ & $\begin{array}{c}2350 \\
2850 \\
\\
840 \\
710 \\
880 \\
260 \\
1320 \\
\text { roup } 1800\end{array}$ & $\begin{array}{c}2710 \\
4140 \\
2660 \\
\\
220 \\
3040 \\
2180 \\
100 \star \star \\
2150\end{array}$ & $\begin{array}{r}240 \\
2670 \\
1790 \\
\\
2340 \\
820 \\
2350 \\
400 \\
1520\end{array}$ & $\begin{array}{r}1480 \\
250 \\
2550 \\
710 \\
1490 \\
330 \\
1450 \\
30 \\
1040\end{array}$ & $\begin{array}{r}3480 \\
280 \\
2060 \\
1670 \\
2860 \\
150 \\
2980 \\
20 \\
1690 \\
\text { Mean }\end{array}$ & $\begin{array}{r}1970 \\
240 \\
1080 \\
1200 \\
1250 \\
30 \\
2490 \\
30 \\
1040 \\
\text { of group }\end{array}$ & $\begin{array}{r}3150 \\
250 \\
1030 \\
870 \\
760 \\
220 \\
200 \\
10 \\
810 \\
1190\end{array}$ & $\begin{array}{r}2390 \\
640 \\
1260 \\
1310 \\
2030 \\
1090 \\
1020 \\
80 \\
1230\end{array}$ & $\begin{array}{r}1150 \\
20 \\
150 \\
680 \\
570 \\
190 \\
340 \\
20 \\
390 \\
\text { Mean }\end{array}$ & $\begin{array}{r}110 \\
90 \\
70 \\
250 \\
530 \\
110 \\
630 \\
20 \\
230 \\
\text { of grou }\end{array}$ & $\begin{array}{r}230 \\
170 \\
0 \\
850 \\
720 \\
0 \\
1090 \\
30 \\
390 \\
440\end{array}$ & $\begin{array}{r}0 \\
330 \\
590 \\
790 \\
1720 \\
670 \\
2600 \\
20 \\
840\end{array}$ & $\begin{array}{r}280 \\
170 \\
670 \\
600 \\
410 \\
100 \\
600 \\
10 \\
360\end{array}$ & $\begin{array}{r}0 \\
30 \\
20 \\
170 \\
50 \\
0 \\
20 \\
0 \\
40 \\
\text { Mean } \\
\text { group }\end{array}$ & $\begin{array}{r}0 \\
0 \\
40 \\
0 \\
60 \\
0 \\
0 \\
0 \\
10 \\
\text { of } \\
30\end{array}$ \\
\hline
\end{tabular}

$\star \star$ There was evidence of an old infarction in the posterior part of the hippocampas on both sides in this brain.

Materials and methods

Samples for this study were taken at necropsy from 16 selected areas of the brain from eight demented subjects whose ages ranged from 70-79 years (table). In every case there was a: history of progressive dementia and a clinical diagnosis of Alzheimer's disease. Pathological diagnosis was based on examination of the formalin-fixed brain in sections prepared from frontal, temporal and parietal lobes, hippocampus, midbrain and pons. Paraffin sections from each of these areas were stained with Cross' modification of the Palmgren stain for neurofibrillary tangles, ${ }^{12}$ NFT, and frozen sections with von Braunmuhl's technique for neuritic plaques. Additional stains employed included luxol fast blue/cresyl violet and the Congo Red stain for amyloid. All cases showed numerous NFT and argyrophilic plaques in hippocampus, frontal and temporal cortex and some NFT in parietal cortex, thus confirming the diagnosis of Alzheimer's disease.

For the purposes of the present study small samples approximately $1 \mathrm{~mm}^{3}$ were removed from the fresh brain from the 16 areas of interest and deep frozen for later estimation of choline acetyl transferase activity (ChAT). The areas chosen for examination (fig 1) were selected as examples of cortex known to have a defined proximity, in terms of their fibre connections, with the entorhinal region, based on comparison with equivalent areas of the monkey brain. ${ }^{1314}$ The areas examined were the following: uncus, amygdala, entorhinal cortex (the anterior part of the parahippocampal gyrus), hippocampus (HI region, mid anteroposterior extent), TH/TF (the posterior part of the parahippocampal gyrus), Brodmann areas 20, 21, 22, 38, 7, 25, 46, 13, 9, 3/1/2/(SI), 17.

Cell free homogenates ${ }^{15}$ were prepared for assay of concentration of total protein ${ }^{16}$ and choline acetyltransferase activity. ${ }^{17}$ Activity of the samples from seven control brains and the eight brains with Alzheimer's disease expressed per $\mathrm{mg}$ of protein were comparable with previously published data from another series. ${ }^{15}$

When the remainder of the brain had been fixed by suspension in neutral, buffered formalin for 3-4 weeks, samples immediately adjacent to those removed for biochemical analysis were dissected and embedded in paraffin wax. Three interrupted $10 \mu \mathrm{m}$ sections at $100 \mu \mathrm{m}$ intervals were cut from each block and stained by the Cross method for NFT. Each section was coded so that the observer was unaware of the case number or site of origin, projected using a Leitz micropromar projection microscope, and camera lucida drawings made of the NFT present in a length of cortex of $2 \mathrm{~mm}$, at a magnification of X80. The counts of the NFT were made on an automatic image analyser (Kentron Image Processing System) using a macroviewer. Mean counts for each area from each case were obtained by averaging the counts obtained from the three sections.
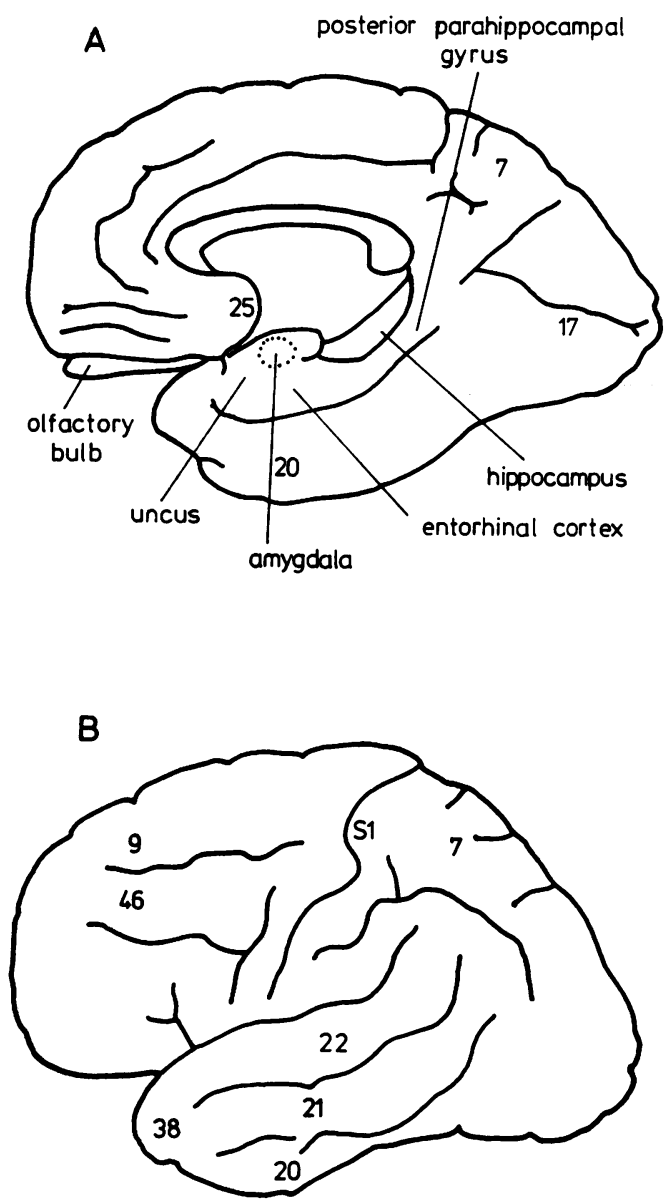

Figure 1 Schematic figure showing the sites that have been sampled for the quantitative study on outlines of the medial $(A)$ and lateral $(B)$ surfaces of the cerebral hemisphere. The numbers indicate the areas of neocortex as delimited by Brodmann and S1 the primary somatic sensory area. Area 13 on the orbital surface of the
hemisphere is not shown. 
Figure 2 To show the distribution and severity of the pathology, as indicated by numbers of neurofibrillary tangles; एolfactory and medial temporal structures with a mean of 1800 tangles / $\mathrm{mm}^{3} ; \mathrm{QZ}$, lateral temporal neocortex, mean 1190 tangles $/ \mathrm{mm}^{3} ; \therefore$, frontal and parietal association cortex, mean 440 tangles $/ \mathrm{mm}^{7}$ and $[\because$ for the minimal involvement of the primary somatic and visual sensory areas.
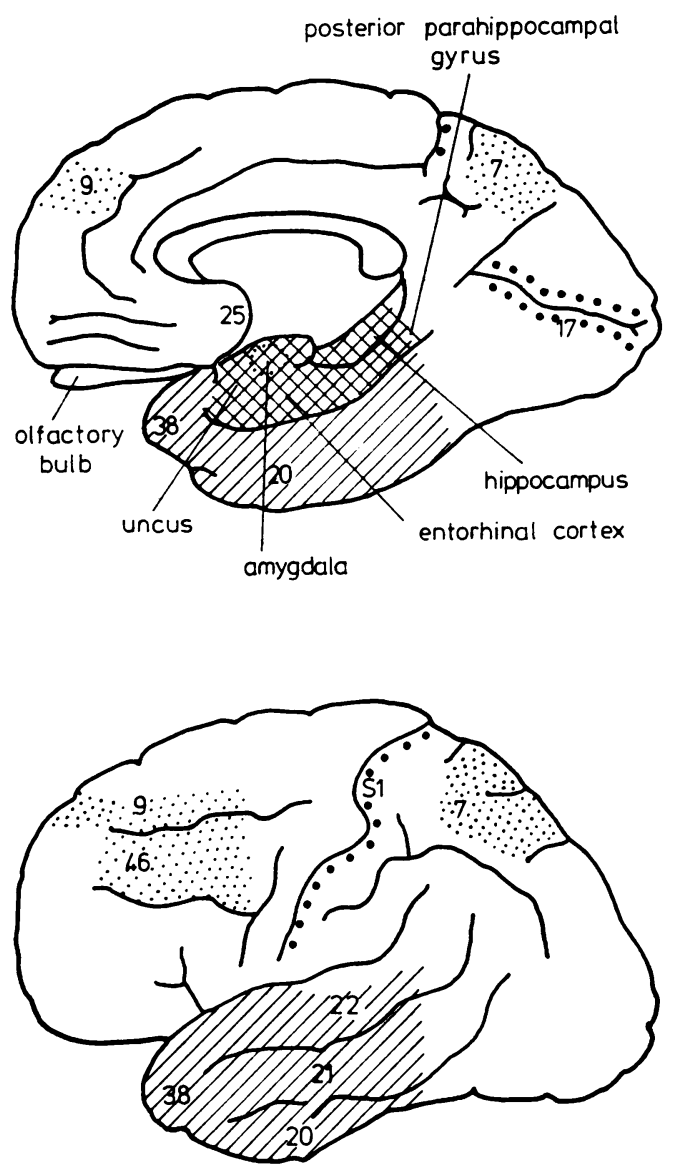

Results

The counts of the neurofibrillary tangles are given in the table, and are summarised in fig 2 . Although there is appreciable variation in the absolute numbers there are certain findings that are reasonably consistent from case to case. The parts of the brain related to the olfactory system, the uncus and the amygdala, and the hippocampus and parahippocampal gyrus are severely involved in all brains, and the uncus and hippocampus have the highest mean counts of tangles. The sites that are next most severely affected are those areas of the neocortex on the lateral surface of the temporal lobe and on the orbital surface of the frontal lobe. In the temporal lobe the highest counts are in areas 20 and 21 , both of which are closely related to the visual system, and in area 38 at the temporal pole; area 7 of the parietal lobe and areas 46 and 9 on the lateral surface of the

Figure 3 The correlation between the mean tangle counts in the four major groups of areas and the mean percentage decrease in ChAT activity in these areas.

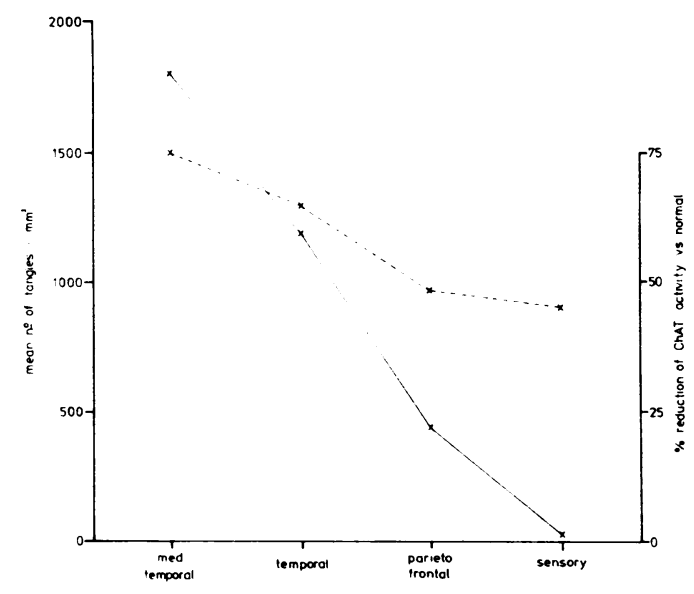

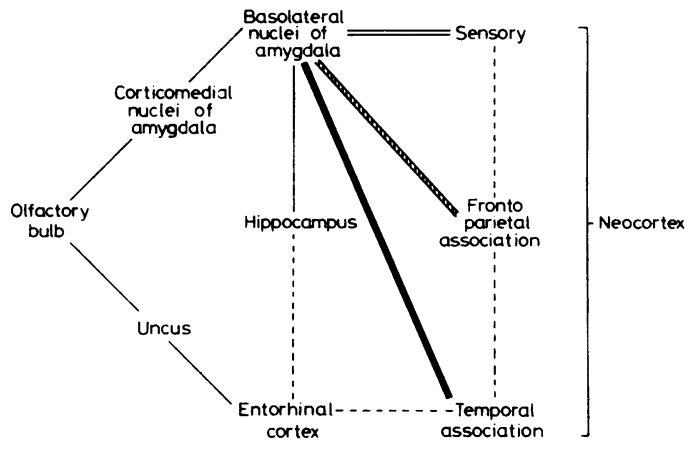

Figure 4 A highly schematic and simplified diagram of the connections between the areas that have been studied here; most of these connections are reciprocal. The connections between the structures in the medial part of the temporal lobe are shown as single lines, those between the amygdala and the neocortex as double lines (the density being indicated by = for few. moderate and $\longrightarrow$ for maximal) and the seyuence of cortico-cortical connections between areas of the neocortex as broken lines.

frontal lobe are approximately the same and are considerably less than the temporal and orbital areas. The primary somatic and visual sensory areas are minimally involved.

The assays of ChAT activity showed a significant decrease in almost all areas examined compared with seven control brains, and the means of the percentage decreases for the different groups of areas correlate well with the counts of the neurofibrillary tangles (fig 3).

\section{Discussion}

In general, the results of the present quantitative study agree with previous reports on the distribution of the pathological changes in the cerebral hemisphere in Alzheimer's disease, ${ }^{1}$ and they also support the hypotheses that the disease process may spread, in either an antegrade or retrograde direction, along a sequence of cortico-cortical connections ${ }^{2}$ or through the fibre connections between the neocortex and amygdala or by both these sets of connections.

The structures in the medial part of the temporal lobe are the most severely affected; the uncus, or olfactory cortex, and the hippocampus, had the highest number of tangles, with the amygdala, the entorhinal cortex and the posterior part of the parahippocampal gyrus (TH/TF) coming next. The high numbers of tangles in the uncus and amygdala may indicate the initial involvement of the olfactory system in the disease, ${ }^{1}$ and indeed it has now been shown that "nasal epithelium tissue taken at autopsy shows unique pathological changes in morphology, distribution and immunoreactivity of neuronal structures in patients with Alzheimer's disease". ${ }^{17 \mathrm{~A}}$ The equally severe involvement of the hippocampus is probably due to the direct connections between it and the amygdala ${ }^{18-21}$ (fig 3) and small numbers of tangles may be present in the uncus, amygdala and hippocampus in the non-demented elderly brain. ${ }^{22-24}$ The higher mean count of tangles (and the greater individual count in 4 of the 6 cases) in the entorhinal cortex in the anterior part of the 
parahippocampal gyrus than in areas $\mathrm{TH} / \mathrm{TF}$ in the posterior part of this gyrus would have been predicted for two reasons; cortico-cortical connections pass from $\mathrm{TH} / \mathrm{TF}$ to the entorhinal corte ${ }^{25}$ which would therefore be a later link in the sequence between the neocortex and the hippocampus, and the entorhinal cortex is more closely related to the olfactory pathway and the amygdala. ${ }^{101125}$

The areas on the lateral surface of the temporal lobe that have been studied here have direct and reciprocal connections with the entorhinal area, ${ }^{4}$ and they form the final steps between the three major sensory systems and the hippocampal formation. ${ }^{3}$ The high number of tangles in all of them may therefore be considered to support the suggestion that the disease process spreads from the structures in the medial temporal lobe to the neocortex along these connections. ${ }^{2}$ Of the other neocortical areas only area 13 on the orbital surface of the frontal lobe, which is similarly interconnected with the entorhinal area ${ }^{26}$ and with area 38 at the temporal pole, ${ }^{3}$ has a comparably high number of tangles. The cortex of area 7 in the parietal lobe and of areas 46 and 9 on the lateral surface of the frontal lobe are only moderately affected, and this may reflect their intermediate position on the sequence of cortico-cortical connections. It would have been expected, from its later position on the sequence of connections, that area 25 on the medial surface would have contained a greater number of tangles than was found.

The minimal involvement of the primary visual and somatic sensory areas agrees with earlier accounts of the distribution of the pathology in this disease ${ }^{1}$ and with the hypothesis that the disease process may spread in a retrograde way along the sequence of corticocortical connections that begins in the sensory areas and ends in the hippocampal formation. ${ }^{2}$

Neither the absolute figures for the neurofibrillary tangles nor the mean numbers should be given unnecessary attention as reflecting the severity of the pathological involvement because there is evidence of loss of nerve cells, particularly in the medial temporal areas ${ }^{27}$ and in the brains of patients with long durations of clinical symptoms. ${ }^{28}$

The numbers of tangles in the different areas of cortex that have been studied here parallel remarkably closely the density of the connections between any individual area and the amygdala. ${ }^{1011}$ Dense connections have been described in experimental studies in the monkey, such as the lateral surface of the temporal lobe, ${ }^{10112930}$ which has the maximum number of tangles, whereas there are sensory areas with few such amygdala-cortical connections ${ }^{1011}$ and minimal number of tangles. It is possible, therefore, that the disease process in Alzheimer's disease may pass along the reciprocal connections between the amygdala and the cortex as well as, or instead of, along the sequence of cortico-cortical connections. It is significant that the areas of cortex with the greatest density of connections with the amygdala and the highest numbers of tangles are also those which are situated late on the series of links between the sensory areas and the hippocampus. The entorhinal cortex, and particularly its anterior part, has strong connections with the amygdala, ${ }^{1011}$ and the high number of tangles here could therefore be related both to these connections and to the projection to it from the olfactory pathway. ${ }^{25}$ The severe involvement of the hippocampus could also have a two-fold basis, the direct projection from the amygdala ${ }^{2021}$ and the major afferent pathway to it from the entorhinal cortex. The cortex in areas TH/TF in the posterior part of the parahippocampal gyrus receives a lighter projection from the amygdala, ${ }^{1011}$ but it does project into the entorhinal cortex. ${ }^{25}$

The correspondence between the density of connections with the amygdala and the degree of pathological change is particularly clear in those areas on the lateral surface of the temporal lobe. Areas 38, 20, 21 and 22 all have dense reciprocal connections with the amygdala ${ }^{10112930}$ and they also have the highest number of tangles of any part of the neocortex. The number of tangles in area 7 of the parietal lobe is substantially less than in the temporal lobe, and this agrees with the quite localised origin and termination of the connection with the amygdala to the part of this area in the depth of the intraparietal sulcus in the monkey. ${ }^{1011}$ In the frontal lobe the modest numbers of tangles in areas 9 and 46 on the lateral surface agrees with the smaller number of fibres from the amygdala to these areas, but the number of tangles is area 25 on the medial surface is less than the number predicted on the basis of the relatively dense projections to it from the amygdala. ${ }^{11}{ }^{11}$ The consistently severe involvement of the amygdala in all the brains and the suggestion that the disease spreads thence to the neocortex agree with two other findings. Marked ChAT deficits have been found in the amygdala in cases of Alzheimer's disease with pathological change but with no significant decrease in ChAT activity in either the neocortex or the hippocampus, ${ }^{31}$ and there is early and severe involvement of the amygdala in Down's syndrome. ${ }^{32}$

Just as the reciprocal connections between the amygdala and the neocortex may be one factor responsible for the distribution of the pathology in the neocortex so also may be the connections of the amygdala with other subcortical structures. Thus the presence of neurofibrillary tangles in such sites as the basal nucleus, the hypothalamus and the raphe nuclei of the brainstem may be attributed to their connections with the amygdala.

The purpose of the ChAT assay was to determine the correlation, in any area, between the structural and biochemical changes. A correlation would be expected if the changes in ChAT activity were due to the secondary degeneration of the cholinergic fibres from the basal nucleus. The ChAT content of the areas which have been studied generally agreed with the degree of pathological change indicated by the number of tangles (fig 2). In the sensory areas the reduction in ChAT activity is clearly shown whereas the tangles are negligible, but it 
is known that senile plaques may be present in these areas, and therefore the ChAT content and plaque formation may represent earlier changes than the tangle formation.

The number of nerve cells through the depth of the cortex is remarkably constant in most areas of the neocortex, and in a $30 \mu \mathrm{m}$ width in a section of $25 \mu \mathrm{m}$ thickness there are approximately 100 nerve cells. ${ }^{34}$ If one assumes the average thickness of the cortex to be $2.0 \mathrm{~mm}$ there are 100 cells in $.025 \mathrm{~mm} \times .03 \mathrm{~mm} \times$ $2 \mathrm{~mm}\left(.0015 \mathrm{~mm}^{3}\right)$, and in $1 / \mathrm{mm}^{3}$ there are approximately 70,000 cells, of which around two-thirds, 45,000 , are pyramidal cells. ${ }^{34}$ The maximum number of tangles that have been found in any area of the neocortex in the present cases is $3,500 / \mathrm{mm}^{3}$, (Case 1, Area 20; table) and these are therefore present in about $10 \%$ of the pyramidal neurons. The cortex of area 7 and of area 46 have been shown to send fibres to at least 15 other cortical areas in the ipsilateral hemisphere and to a number of subcortical structures such as the striatum, claustrum, thalamus, tectum and pons. ${ }^{35}$ The population of 45,000 pyramidal neurons is therefore projecting to 20 other sites, at least, with an average of $5 \%$ of them to each site. As about $10 \%$ of these cells may be affected by tangles in Alzheimer's disease, is it too speculative to suggest that these are the cells of origin of two major efferent pathways, one cortico-cortical to the medial temporal cortex and the other cortico-subcortical to the amygdala?

We thank Miss Ann Stanmore and Mr R N L Brooke of the Department of Human Anatomy, Oxford, Miss Stanmore for counting the neurofibrillary tangles with the Image Processing System and Mr Brooke for drawing the figures. This work was supported by grants from the Medical Research Council and the Wellcome Trust.

1 Pearson RCA, Powell TPS. The neuroanatomy of Alzheimer's disease. Rev Neurosci 1989 (in press).

2 Pearson RCA, Esiri MM, Hiorns RW, Wilcock GK, Powell TPS. Anatomical correlates of the distribution of the pathological changes in the neocortex in Alzheimer's disease. Proc Natl Acad Sci USA 1985;82:4531-4.

3 Jones EG, Powell TPS. An anatomical study of converging sensory pathways within the cerebral cortex of the sensory pathways within the
monkey. Brain 1970;93:793-820.

4 Kosel KG, Van Hoesen GW, Rosene DL. Non-hippocampal cortical projections from the entorhinal cortex in campal cortical projections from the entorhinal cortex in
the rat and rhesus monkey. Brain Res 1982;244:201-13.

5 Van Hoesen GW. The parahippocampal gurus: new an Hoesen GW. The parahippocampal gurus: new
observations regarding its cortical connections in the monkey. Trends Neurosci 1982:5:345-50.

6 Powell TPS, Cowan WM, Raisman G. The central olfactory connections. J Anat 1965;99:791-813.

7 Krettek JE, Price JL. A description of the amygdaloid complex in the rat and cat with observations on intraamygdaloid axonal connections. J Comp Neurol 1978; 178:255-80.

8 Ottersen DP. Connections of the amygdala of the rat. IV Cortico amygdaloid and intraamygdaloid connections as studied with axonal transport of horseradish peroxsidase. J Comp Neurol 1982;205:30-48.
9 Aggleton JP. A description of intra-amygdaloid connections in old world monkeys. Exp Brain Research 1985;57:390-9.

10 Aggleton JP, Burton MJ, Passingham RE. Cortical and subcortical afferents to the amygdala of the rhesus monkey subcortical afferents to the amygdala of the rhesus mo
(Macaca mulatta). Brain Research 1980;190:347-68.

11 Amaral DG, Price JL. Amygdalo-cortical projections in the monkey (Macaca fascicularis). J Comp Neurol 1984; 230:465-96

12 Cross RB. Demonstration of neurofibrillary tangles in paraffin section-a quick and simple method using Palmgren's technique. Med Lab Sci 1982;39:67-9.

13 Brodman K. Vergleichende Lokalisationslehre der Grosshumrinde in ihren Prinzipien dargestellt auf Grand des Zellenbaues Leipzig: Barth, 1909.

14 Bonin G, von Bailey P. The neocortex of Macaca Mulatta. Urbana: Univ Illinois Press, 1947.

15 Procter AW, Lowe SL, Palmer AM, et al. Topographical distribution of neurochemical changes in Alzheimer's disease. J Neurol Sci 1988;84:125-40.

16 Lowry OH, Rosebrough NJ, Farr AL, Randall RJ. Protein measurement with the Folin phenol reagent. J Biol Chem measurement with

17 Fonnum F. A rapid radiochemical method for the determination of choline acetyltransferase activity. $J$ Neurochem 1975;24:407-9.

17A Talamo BR, Rudel RA, Kosik KS, et al. Pathological changes in olfactory neurons in patients with Alzheimer's disease. Nature 1989;337:736-9.

18 Rosene DL, Van Hoesen GW. Hippocampal efferents reach widespread areas of cerebral cortex and amygdala in the rhesus monkey. Science 1977;198:315-7.

19 Kretteck JE, Price JL. Projections from the amygaloid complex and adjacent olfactory structures to the entorhinal cortex and to the subiculum in the rat and cat. J Comp Neurol 1977;172:723-52.

20 Amaral DG, Cowan WM. Subcortical afferents to the hippocampal formation in the monkey. J Comp Neurol 1980;189:573-91.

21 Aggleton JP. A description of the amygdalo-hippocampal interconnections in the macaque monkey. Exp Brain Res interconnections

$1986 ; 64: 515-26$.
Ulrich J. Senile plaques and neurofibrillary tangles of the Alzheimer type in nondemented individuals at presenile age. Gerontol 1982;28:86-90.

23 Ulrich J. Alzheimer changes in nondemented subjects younger than sixty five: possible early stages of Alzheimer's disease and senile dementia of Alzheimer type. Ann Neurol 1985;17:273-7.

24 Mann DMA, Tucker CM, Yates PO. The topographic distribution of senile plaques and neurofibrillary tangles in the brains of non-demented persons of different ages. Neuropathol Appl Neurobiol 1987;13:123-39.

25 Van Hoesen GW, Pandya DN. Some connections of the entorhinal area (area 28) and perirhinal area (area 35) cortices of the rhesus monkey. I Temporal lobe afferents. Brain Res 1975;95:1-24.

26 Van Hoesen GW, Pandya DN, Butters N. Some connections of the entorhinal (area 28) and perirhinal (area 35) cortices of the entorhinal (area 28) and perirhinal (area 35) cortices
of the rhesus monkey. II Frontal lobe afferents. Brain Res of the rhesus monk
$1975 ; 95: 25-38$.

27 Hyman BT, Van Hoesen GW, Damasio AR, Barnes CL. Alzheimer's disease: cell specific pathology isolates the hippocampal formation. Science 1984;225:1168-70.

28 Mann DMA, Marcyniuk B, Yates PO, Neary D, Snowden JS. The progression of the pathological changes of Alzheimer's disease in frontal and temporal neocortex examined both at biopsy and at autopsy. Neuropathol $A p p$ Neurobiol 1988;14:177-95.

29 Herzog AG, Van Hoesen GW. Temporal neocortical afferent connections to the amygdala in the rhesus monkey. Brain Res 1976;115:57-69.

30 Turner BH, Mishkin M, Knapp M. Organization of the amygdalopetal projections from modality-specific cortical association areas in the monkey. J Comp Neurol association areas
1980;191:515-43.

31 Palmer AM, Proctor AW, Stratmann GL, Bowen DM. Exitatory amino acid-releasing and cholinergic neurones
in Alzheimer's disease. Neurosci Lett 1986;66:199-204.

32 Mann DA, Esiri MM. The pattern of acquisition of plaques and tangles in the brains of patients under 50 years of age with Down's syndrome. J Neurol Sci 1989;89:169-79.

33 Wilcock GK, Esiri MM, Bowen DM, Smith CCT Alzheimer's disease. Correlations of cortical choline acetyl transferase activity with the severity of dementia and histological abnormalities. J Neurol Sci 1982;57:407-17.

34 Rockel AJ, Hiorns RW, Powell TPS. The basic uniformity of the cerebral cortex. Brain 1980;103:221-44.

35 Selemon LD. Goldman-Rakic PS. Common cortical and subcortical targets of the dorsolateral prefrontal and posterior parietal cortices in the rhesus monkey; evidence for a distributed network subserving spatially guided behaviour. J Neurosci 1988;8:4049-68. 